\title{
Tolerance analysis for 0-1 knapsack problems
}

\author{
Pisinger, David; Saidi, Alima
}

Published in:

European Journal of Operational Research

Link to article, DOI:

10.1016/j.ejor.2016.10.054

Publication date:

2017

Document Version

Peer reviewed version

Link back to DTU Orbit

\section{Citation (APA):}

Pisinger, D., \& Saidi, A. (2017). Tolerance analysis for 0-1 knapsack problems. European Journal of Operational Research, 258(3), 866-876. https://doi.org/10.1016/j.ejor.2016.10.054

\section{General rights}

Copyright and moral rights for the publications made accessible in the public portal are retained by the authors and/or other copyright owners and it is a condition of accessing publications that users recognise and abide by the legal requirements associated with these rights.

- Users may download and print one copy of any publication from the public portal for the purpose of private study or research.

- You may not further distribute the material or use it for any profit-making activity or commercial gain

- You may freely distribute the URL identifying the publication in the public portal

If you believe that this document breaches copyright please contact us providing details, and we will remove access to the work immediately and investigate your claim. 


\title{
Tolerance analysis for 0-1 knapsack problems
}

\author{
David Pisinger ${ }^{1}$, Alima Saidi ${ }^{2}$ \\ ${ }^{1}$ DTU Management, Technical University of Denmark, \\ phone: +45 45254555, email: pisinger@man.dtu.dk \\ ${ }^{2}$ DIKU, University of Copenhagen
}

October 31, 2016

\begin{abstract}
Post-optimal analysis is the task of understanding the behavior of the solution of a problem due to changes in the data. Frequently, post-optimal analysis is as important as obtaining the optimal solution itself. Post-optimal analysis for linear programming problems is well established and widely used. However, for integer programming problems the task is much more computationally demanding, and various approaches based on branch-and-bound or cutting planes have been presented. In the present paper we study how much coefficients in the original problem can vary without changing the optimal solution vector, the so-called tolerance analysis. We show how to perform exact tolerance analysis for the 0-1 knapsack problem with integer coefficients in amortized time $O(c \log n)$ for each item, where $n$ is the number of items, and $c$ is the capacity of the knapsack. Amortized running times report the time used for each item, when calculating tolerance limits of all items. Exact tolerance limits are the widest possible intervals, while approximate tolerance limits may be suboptimal. We show how various upper bounds can be used to determine approximate tolerance limits in time $O(\log n)$ or $O(1)$ per item using the Dantzig bound and Dembo-Hammer bound, respectively. The running times and quality of the tolerance limits of all exact and approximate algorithms are experimentally compared, showing that all tolerance limits can be found in less than a second. The approximate bounds are of good quality for large-sized instances, while it is worth using the exact approach for smaller instances.
\end{abstract}

Keywords: Robustness \& Sensitivity Analysis; Knapsack Problem; Post-optimal Analysis; Dynamic Programming

\section{Introduction}

In many combinatorial optimization problems the data are not given with certainty, and hence a natural question is how large the errors on the coefficients can be without distorting the sought optimal solution. Combinatorial problems, unlike linear programming problems, behave in an 
unstable manner under small changes in the initial data, making tolerance analysis a challenging but important problem.

In this paper we distinguish between sensitivity analysis and tolerance analysis. Sensitivity analysis in linear programming studies in which range the coefficients can vary without changing the current basic solution. Since we do not have basic solutions in combinatorial problems, tolerance analysis studies the robustness of an optimal solution vector to perturbations in the problem coefficients. Tolerance analysis is also known as stability analysis in the literature.

Greenberg [7] gives a quite recent bibliography for post-optimal analysis in combinatorial optimization, and mentions a number of papers on knapsack problems $[4,8,14,22]$. Klein and Holm [13] presented a general cutting-plane framework for post-optimal analysis of combinatorial problems and gave sufficient conditions for preserving the same optimal solution when the right-hand side or an objective coefficient is altered.

The 0-1 knapsack problem consists of packing a subset of $n$ items, each item $i$ having a profit $p_{i}$ and a weight $w_{i}$, into a knapsack of capacity $c$ such that the overall profit is maximized. See, e.g., Kellerer et al. [12] for a thorough introduction. Tolerance analysis for the knapsack problem consists of determining the intervals $\alpha_{p_{k}} \leq p_{k} \leq \beta_{p_{k}}$ and $\alpha_{w_{k}} \leq w_{k} \leq \beta_{w_{k}}$ for which the profit or the weight of a given item $k$ can be perturbed such that a given optimal solution remains optimal for the problem. Exact tolerance limits are the widest possible intervals, while approximate tolerance limits may be suboptimal (i.e., a subset of the exact tolerance limits). Notice that at any time we only alter a single item $k$.

Hifi et al. [11] proved several results that characterize the tolerance limits. Using these results they proposed two algorithms, one to compute the profit tolerances and one to compute the weight tolerances. The profit algorithm, having a running time of $O\left(n^{2}\right)$, applies upper bounds to derive exact and approximate tolerance intervals. The weight algorithm, having a running time of $O\left(n^{2} c\right)$, applies dynamic programming to derive exact and approximate tolerance intervals.

The main objective of this paper is to present an exact algorithm for the tolerance analysis of the $0-1$ knapsack problem based on dynamic programming. The algorithm can determine the exact tolerance interval for the profit or weight of an arbitrary item.

This approach resembles the approach of Hifi et al. [11] in the way that both approaches take advantage of the dynamic programming solution, but differs in the fact that some of the results of Hifi et al. [11] are approximate while this new method is exact for all results. In addition the new algorithm has a better computation time, $O(n c \log n)$.

Table 1 summarizes the results of Hifi et al. [11] and of the present paper, reporting the time needed to compute a tolerance limit for a specific item $k$. The first two rows concern the perturbation of profit $p_{k}$ while the next two rows concern the perturbation of weight $w_{k}$. Columns 4, 6 and 7 report running times for finding Exact tolerance limits, while columns 5, 8 and 9 report running times for finding Approximate tolerance limits. Depending on the value of the current optimal solution $x_{k}^{*}$ the upper and lower limits can be calculated in a variety of ways. All running times are for a given item $k$, and it is assumed that the current optimal solution is known in advance, including the residual capacity of the solution. Worstcase denotes worst-case running time, while Amortized denotes amortized running time. Amortized running times report the time used for each item, when calculating tolerance limits of all items. Two different approximate 
Table 1: Summary of the results presented by Hifi et al. [11] and the present paper. Notice that the quality of the approx bounds is different. The approx LP-bound generally gives the most correct tolerance limits of the three approx methods.

\begin{tabular}{|c|c|c|c|c|c|c|c|c|}
\hline \multirow{2}{*}{$\begin{array}{l}\text { Pertur- } \\
\text { bation }\end{array}$} & \multirow{2}{*}{$\begin{array}{c}\text { Current } \\
\text { Solution } \\
x^{*} \\
\end{array}$} & \multirow[t]{2}{*}{ Limit } & \multicolumn{2}{|c|}{ Hifi et al. [11] } & \multicolumn{4}{|c|}{ Our Results } \\
\hline & & & Exact & Approx & $\begin{array}{c}\text { Exact } \\
\text { Worstcase }\end{array}$ & $\begin{array}{c}\text { Exact } \\
\text { Amortized }\end{array}$ & $\begin{array}{c}\text { Approx } \\
\text { LP-bound }\end{array}$ & $\begin{array}{c}\text { Approx } \\
\text { DH-bound }\end{array}$ \\
\hline \multirow[t]{2}{*}{ profit $p_{k}$} & 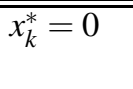 & $\begin{array}{l}\alpha_{p_{k}} \\
\beta_{p_{k}}\end{array}$ & $O(1)$ & $O(n)$ & $\begin{array}{l}O(1) \\
O(n c)\end{array}$ & $\begin{array}{l}O(1) \\
O(c \log n)\end{array}$ & $O(\log n)$ & $O(1)$ \\
\hline & $x_{k}^{*}=1$ & $\begin{array}{l}\alpha_{p_{k}} \\
\beta_{p_{k}}\end{array}$ & $O(1)$ & $O(n)$ & $\begin{array}{l}O(n c) \\
O(1)\end{array}$ & $\begin{array}{l}O(c \log n) \\
O(1)\end{array}$ & $O(\log n)$ & $O(1)$ \\
\hline \multirow[t]{2}{*}{ weight $w_{k}$} & $x_{k}^{*}=0$ & $\begin{array}{l}\alpha_{w_{k}} \\
\beta_{w_{k}}\end{array}$ & $O(1)$ & $\overline{O\left(n^{2} c\right)}$ & $\begin{array}{l}O(n c) \\
O(1)\end{array}$ & $\begin{array}{l}O(c \log n) \\
O(1)\end{array}$ & $O(\log n)$ & $\overline{O O(1)}$ \\
\hline & $x_{k}^{*}=1$ & $\begin{array}{l}\alpha_{w_{k}} \\
\beta_{w_{k}}\end{array}$ & $O(n)$ & $O\left(n^{2} c\right)$ & $\begin{array}{l}O(n c) \\
O(n)\end{array}$ & $\begin{array}{l}O(c \log n) \\
O(1)\end{array}$ & $O(\log n)$ & $O(1)$ \\
\hline
\end{tabular}

tolerance limits are presented in this paper using either the Dantzig upper bound (Approx LPbound) or Dembo-Hammer [5] upper bound (Approx DH-bound).

Several related problems have been studied recently in the literature: Belgacem and Hifi [3] and [10] consider the perturbation of a subset of items in a binary knapsack problem. Monaci et al. [18] consider the related robust knapsack problem. Archetti et al. [1] consider the reoptimization of a knapsack problem when new items are added to the problem. Various heuristics and approximation algorithms are presented. Monaci and Pferschy [17] consider a variant of the knapsack problem where the exact weight of each item is not known in advance but belongs to a given interval. The worsening of the optimal solution is analyzed. Plateau and Plateau [21] consider how a knapsack problem can be reoptimized given that the data has been slightly modified.

The paper is organized as follows: Section 2 describes the 0-1 knapsack problem and its "dual" denoted the weight knapsack problem, which is advantageous when determining weight tolerance limits. Dynamic programming methods and upper/lower bounds are presented for both problems. Section 3 formally defines the tolerance analysis of a 0-1 knapsack problem and presents some special cases for which the profit or weight tolerance limits can be identified. Section 4 presents the exact profit and weight tolerance limits, and describes an $O(n c)$ algorithm per item (or $O\left(n^{2} c\right)$ in total) which can be used to calculate the limits. Section 5 shows how the amortized time complexity of the algorithm can be improved to $O(c \log n)$ per item (or $O(n c \log n)$ in total) by making use of overlapping subproblems in the dynamic programming. Moreover, we show how to calculate the tolerance limits by solving a single 0-1 knapsack problem. This makes it possible to use any state-of-the-art algorithm for solving the knapsack problem, and introduces the opportunity to find approximate tolerance limits by use of various upper bounds for the $0-1$ knapsack problem. 


\section{The 0-1 Knapsack Problem}

The 0-1 knapsack problem consists of packing a subset of $n$ items into a knapsack of capacity $c$. Each item $i$ has profit $p_{i}$ and weight $w_{i}$ and the objective is to maximize the profit of the items in the knapsack without exceeding the capacity $c$. Using the binary variable $x_{i}$ to indicate whether item $i$ is included in the knapsack, we get the formulation:

$$
\begin{aligned}
\text { (KP) maximize } & \sum_{i=1}^{n} p_{i} x_{i} \\
\text { subject to } & \sum_{i=1}^{n} w_{i} x_{i} \leq c \\
& x_{i} \in\{0,1\}, i=1,2, \ldots, n
\end{aligned}
$$

Without loss of generality we assume that the profits and the weights are positive integers (see Kellerer et al. [12] for transformations to this form). Also, we assume that $\sum_{i=1}^{n} w_{i}>c$. An optimal solution vector to KP is denoted $x^{*}$ and the optimal solution value $z^{*}$. A knapsack problem with capacity $c$ is denoted $\mathrm{KP}[c]$, and we use the terminology $\mathrm{KP}:=\mathrm{KP}[c]$ whenever the capacity is the original capacity. $\mathrm{KP}[c] \backslash\{k\}$ denotes the knapsack subproblem $\mathrm{KP}[c]$ where item $k$ is excluded. $z(K)$ is the optimal objective function of knapsack instance $K . \operatorname{KP}\left(x^{\prime}\right)$ is the instance with variables $x$ fixed at $x^{\prime}$, hence $z\left(\operatorname{KP}\left(x^{\prime}\right)\right)=\sum_{i=1}^{n} p_{i} x_{i}^{\prime}$.

The $L P$-relaxed (or fractional) knapsack problem, where $0 \leq x_{i} \leq 1$ for $i=1,2, \ldots, n$ can be solved to optimality by a greedy algorithm, in which the items are sorted according to nonincreasing profit-to-weight ratio $p_{i} / w_{i}$ and the knapsack is packed with items $1,2, \ldots$ until the first item $s$ (the split item) which does not fit into the knapsack. The optimal solution value $z_{L P}^{*}$ is then

$$
z_{L P}^{*}=\sum_{i=1}^{s-1} p_{i}+\left(c-\sum_{i=1}^{s-1} w_{i}\right) \frac{p_{s}}{w_{s}} .
$$

Knowing that all profits are integers, we may round down the solution value to $\left\lfloor z_{L P}^{*}\right\rfloor$ getting the Dantzig upper bound.

The 0-1 knapsack problem can be solved by use of dynamic programming. Let KP be a knapsack instance, and consider for $j=0, \ldots, n$ the subproblem $\mathrm{KP}_{j}[d]$ of $\mathrm{KP}$ consisting of the items $\{1,2, \ldots, j\}$ and having integer capacity $d \leq c$.

$$
\mathrm{KP}_{j}[d]=\max \left\{\sum_{i=1}^{j} p_{i} x_{i} \mid \sum_{i=1}^{j} w_{i} x_{i} \leq d ; x_{i} \in\{0,1\}, \forall i\right\}=\mathrm{KP}[d] \backslash\{j+1, j+2, \ldots, n\} .
$$

Let the optimal solution value of $\mathrm{KP}_{j}[d]$ be denoted $z_{j}(d)$. The values of $z_{j}(d)$ can be calculated by use of the following recursion:

$$
z_{j}(d)=\max \left\{z_{j-1}(d), z_{j-1}\left(d-w_{j}\right)+p_{j}\right\},
$$

where we set $z_{0}(d)=0$ for $d=0, \ldots, c$. We assume that $z_{j-1}\left(d-w_{j}\right)=-\infty$ when $d-w_{j}<0$. The running time of Recursion (4) is $O(n c)$. If we only save undominated states in the dynamic programming recursion (i.e. pairs of $\left(d, z_{j}(d)\right)$ which do not dominate each other) the running time can be limited to $O\left(n \min \left\{c, z^{*}\right\}\right)$, where $z^{*}$ is the optimal solution value of KP. 


\subsection{Weight Knapsack Problem}

The 0-1 knapsack problem has a reverse formulation

$$
\begin{array}{rll}
(\mathrm{WKP}) & \text { minimize } & \sum_{i=1}^{n} w_{i} x_{i} \\
\text { subject to } & \sum_{i=1}^{n} p_{i} x_{i} \geq z \\
& x_{i} \in\{0,1\}, i=1,2, \ldots, n
\end{array}
$$

where we ask for the minimum weight sum such that the profit sum $z$ can be achieved. A specific weight knapsack problem with target sum $z$ will be denoted $\mathrm{WKP}[z] \operatorname{WKP}[z] \backslash\{k\}$ denotes the weight knapsack subproblem WKP $[z]$ where item $k$ is excluded. $y(K)$ is the optimal objective function of weight knapsack instance $K$. $\mathrm{WKP}\left(x^{\prime}\right)$ is the instance with variables $x$ fixed at $x^{\prime}$, hence $y\left(\operatorname{WKP}\left(x^{\prime}\right)\right)=\sum_{j=1}^{n} w_{j} x_{j}^{\prime}$.

If $\mathrm{KP}[c]$ has a unique optimal solution $x^{*}$ with solution value $z^{*}$ then $x^{*}$ will also be a unique optimal solution to $\mathrm{WKP}\left[z^{*}\right]$. If several equivalent solutions to $\mathrm{KP}[c]$ exist with the solution value $z^{*}$ then $\mathrm{WKP}\left[z^{*}\right]$ will return a solution using the least weight.

The $L P$-relaxed (or fractional) weight knapsack problem, where $0 \leq x_{i} \leq 1$ for $i=1,2, \ldots, n$ can be solved in a similar way as the ordinary knapsack problem by sorting the items according to nonincreasing profit-to-weight ratio. Let the weight split item $s^{\prime}$ be the first item where the profit sum is not smaller than $z$. The optimal solution value $z_{L P}^{w *}$ is then

$$
z_{L P}^{w *}=\sum_{i=1}^{s^{\prime}-1} w_{i}+\left(z-\sum_{i=1}^{s^{\prime}-1} p_{i}\right) \frac{w_{s^{\prime}}}{p_{s^{\prime}}} .
$$

Knowing that all weights are integers, we may round up the solution value to $\left\lceil z_{L P}^{w *}\right\rceil$ getting what we will call the weight Dantzig lower bound.

We may solve WKP by use of dynamic programming in time $O\left(n \min \left\{c, z^{*}\right\}\right)$, where $z^{*}$ is the optimal solution value of KP (see Section 3.4 in [12]).

Notice, that a weight knapsack problem WKP can be transformed to an ordinary knapsack problem KP as follows. Let the total profit and weight be given as $p^{T}=\sum_{i=1}^{n} p_{i}$ and $w^{T}=\sum_{i=1}^{n} w_{i}$. Then we have

$$
\begin{aligned}
y(\mathrm{WKP}[z]) & =\min \left\{\sum_{i=1}^{n} w_{i} x_{i} \mid \sum_{i=1}^{n} p_{i} x_{i} \geq z ; x_{i} \in\{0,1\}, i=1,2, \ldots, n\right\} \\
& =w^{T}-\max \left\{\sum_{i=1}^{n} w_{i} x_{i} \mid \sum_{i=1}^{n} p_{i} x_{i} \leq p^{T}-z ; x_{i} \in\{0,1\}, i=1,2, \ldots, n\right\} .
\end{aligned}
$$

Hence we may use an ordinary KP algorithm for solving the latter maximization problem. 


\section{Tolerance Analysis}

Let KP be a knapsack instance with an optimal solution $x^{*}$ and an optimal solution value $z^{*}$. If more than one optimal solution exists, we will in the following assume that the solution $x^{*}$ with the least weight sum is chosen (i.e., with the largest residual capacity). Notice that this is an important assumption since otherwise the stated theorems do not hold. Exact algorithms based on dynamic programming methods can easily be modified to satisfy the property.

Tolerance analysis for the knapsack problem consists of determining the intervals for which the profit or weight of a selected item $k$ can be perturbed such that $x^{*}$ remains an optimal (but not necessarily unique) solution.

Let $\mathrm{KP}_{\Delta p_{k}}$ be the knapsack instance derived from $\mathrm{KP}$ when a single profit $p_{k}$ is substituted with $p_{k}+\Delta p_{k}$ for some $\Delta p_{k} \in \mathbb{Z}$.

$$
\begin{array}{rll}
\left(\mathrm{KP}_{\Delta p_{k}}\right) \text { maximize } & \sum_{i=1}^{n} p_{i} x_{i}+\Delta p_{k} x_{k} \\
\text { subject to } & \sum_{i=1}^{n} w_{i} x_{i} \leq c \\
& x_{i} \in\{0,1\}, i=1,2, \ldots, n
\end{array}
$$

Let $z_{\Delta p_{k}}^{*}$ be the optimal solution value to $\mathrm{KP}_{\Delta p_{k}}$ and $\mathrm{KP}_{\Delta p_{k}}\left(x^{*}\right)=\sum_{i=1}^{n} p_{i} x_{i}^{*}+\Delta p_{k} x_{k}^{*}$ the solution value of the original solution $x^{*}$ in $\mathrm{KP}_{\Delta p_{k}}$. Then we define $\alpha_{p_{k}}$ and $\beta_{p_{k}}$ to be the lower and upper, respectively, tolerance limit of $p_{k}$ in KP as:

$$
\begin{aligned}
\alpha_{p_{k}} & =\min _{\Delta p_{k} \leq 0}\left\{p_{k}+\Delta p_{k} \mid \mathrm{KP}_{\Delta p_{k}}\left(x^{*}\right)=z_{\Delta p_{k}}^{*}\right\}, \\
\beta_{p_{k}} & =\max _{\Delta p_{k} \geq 0}\left\{p_{k}+\Delta p_{k} \mid \mathrm{KP}_{\Delta p_{k}}\left(x^{*}\right)=z_{\Delta p_{k}}^{*}\right\} .
\end{aligned}
$$

Notice that since all coefficients are assumed to be integers, $\alpha_{p_{k}}, \beta_{p_{k}}$ will also be integers.

Analogously, we can define the knapsack problem $\mathrm{KP}_{\Delta w_{k}}$, where a single weight $w_{k}$ is substituted with $w_{k}+\Delta w_{k}$ for some $\Delta w_{k} \in \mathbb{Z}$.

$$
\begin{array}{rll}
\left(\mathrm{KP}_{\Delta w_{k}}\right) \text { maximize } & \sum_{i=1}^{n} p_{i} x_{i} \\
\text { subject to } & \sum_{i=1}^{n} w_{i} x_{i}+\Delta w_{k} x_{k} \leq c \\
& x_{i} \in\{0,1\}, i=1,2, \ldots, n
\end{array}
$$

Let $z_{\Delta w_{k}}^{*}$ be the optimal solution value to $\mathrm{KP}_{\Delta w_{k}}$ and $\mathrm{KP}_{\Delta w_{k}}\left(x^{*}\right)=\sum_{i=1}^{n} p_{i} x_{i}^{*}=z^{*}$ be the solution value of the original solution $x^{*}$ in $\mathrm{KP}_{\Delta w_{k}}$. Then we define $\alpha_{w_{k}}$ and $\beta_{w_{k}}$ to be the lower and upper, respectively, tolerance limit of $w_{k}$ in KP as:

$$
\begin{aligned}
& \alpha_{w_{k}}=\min _{\Delta w_{k} \leq 0}\left\{w_{k}+\Delta w_{k} \mid z^{*}=z_{\Delta w_{k}}^{*}, \sum_{i=1}^{n} w_{i} x_{i}^{*}+\Delta w_{k} x_{k}^{*} \leq c\right\}, \\
& \beta_{w_{k}}=\max _{\Delta w_{k} \geq 0}\left\{w_{k}+\Delta w_{k} \mid z^{*}=z_{\Delta w_{k}}^{*}, \sum_{i=1}^{n} w_{i} x_{i}^{*}+\Delta w_{k} x_{k}^{*} \leq c\right\} .
\end{aligned}
$$


As before we notice that $\alpha_{w_{k}}, \beta_{w_{k}}$ will be integers. KP.

The intervals $\left[\alpha_{p_{k}}, \beta_{p_{k}}\right]$ and $\left[\alpha_{w_{k}}, \beta_{w_{k}}\right]$ thus represent the tolerance intervals for $p_{k}$ and $w_{k}$ in

The naïve way to compute the profit tolerance interval $\left[\alpha_{p_{k}}, \beta_{p_{k}}\right]$ of an item $k$ is to decrease (or increase) by one unit the profit $p_{k}$ until the given optimal solution $x^{*}$ is no longer an optimal, feasible solution. The weight tolerance interval $\left[\alpha_{w_{k}}, \beta_{w_{k}}\right]$ is computed similarly. If we use the dynamic programming Recursion (4) to solve each instance of KP, the overall running time for calculating the tolerance intervals of item $k$ becomes $O\left(n c\left(\beta_{p_{k}}-\alpha_{p_{k}}+\beta_{w_{k}}-\alpha_{w_{k}}\right)\right)$. This can be slightly improved by using binary search, but still the running time may become unacceptably large.

Hifi et al. [11]) identified some special cases in which the tolerance intervals can be calculated easily. These results are summed up in Appendix B.

Table 2: Exact and approximate tolerance limits for an instance with $c=9$ specified in Columns 2 and 3.

\begin{tabular}{|c|rrr|rr|rr|rr|rr|}
\hline Item & \multicolumn{2}{|c|}{$\begin{array}{c}\text { Parameters } \\
\text { and }\end{array}$} & \multicolumn{2}{c|}{$\begin{array}{c}\text { Exact } \\
\text { profit } \\
\text { intervals }\end{array}$} & \multicolumn{2}{|c|}{$\begin{array}{c}\text { Approximate } \\
\text { profit } \\
\text { intervals [11] }\end{array}$} & \multicolumn{2}{|c|}{$\begin{array}{c}\text { Exact } \\
\text { weight } \\
\text { intervals }\end{array}$} & \multicolumn{2}{|c|}{$\begin{array}{c}\text { Approximate } \\
\text { weight } \\
\text { intervals [11] }\end{array}$} \\
\hline$i$ & $w_{i}$ & $p_{i}$ & $x_{i}^{*}$ & $\alpha_{p_{i}}$ & $\beta_{p_{i}}$ & $\alpha_{p_{i}}$ & $\beta_{p_{i}}$ & $\alpha_{w_{i}}$ & $\beta_{w_{i}}$ & $\alpha_{w_{i}}$ & $\beta_{w_{i}}$ \\
\hline 1 & 2 & 6 & 1 & 4 & $\infty$ & 4 & $\infty$ & 2 & 2 & 2 & 2 \\
2 & 3 & 5 & 0 & 0 & 6 & 0 & 4 & 3 & $\infty$ & 3 & $\infty$ \\
3 & 6 & 8 & 0 & 0 & 9 & 0 & 8 & 5 & $\infty$ & 5 & $\infty$ \\
4 & 7 & 9 & 1 & 8 & $\infty$ & 10 & $\infty$ & 5 & 7 & 7 & 7 \\
5 & 5 & 6 & 0 & 0 & 9 & 0 & 6 & 5 & $\infty$ & 5 & $\infty$ \\
6 & 9 & 7 & 0 & 0 & 15 & 0 & 15 & 5 & $\infty$ & 5 & $\infty$ \\
7 & 4 & 3 & 0 & 0 & 4 & 0 & 4 & 2 & $\infty$ & 2 & $\infty$ \\
\hline
\end{tabular}

Table 2 shows the exact profit and weight tolerance intervals computed by the naïve algorithm for a given example. It also lists the approximate tolerance intervals derived when using ApproxLP, the method described in [11]. The tolerance analysis guarantees that the solution remains optimal but not necessarily unique within the found interval. For item $k=3$ we have that $x^{*}$ remains optimal when $p_{3} \in[0,9]$. However, for $p_{3}=9$ we have two optimal solutions: $x^{*}=(1,0,0,1,0,0,0)$ or $x^{*}=(1,0,1,0,0,0,0)$ both with $z^{*}=15$.

\section{Exact Tolerance Analysis}

In this section we present necessary and sufficient criteria for $x^{*}$ to remain optimal under various perturbations of item $k$. The analysis makes use of dynamic programming, where we in turn place the studied item $k$ as the last item, in order to state the optimality criteria. An illustrative example is presented in Appendix A. Stages in the dynamic programming recursion (3) correspond to the addition of one item (i.e. one column in the dynamic programming table), while states 
correspond to the individual values in the table (i.e. a capacity $d$ and the solution $z_{j}(d)$ ). Instead of writing a state as a pair $\left(d, z_{j}(d)\right)$, we will often use the shorthand notation $z_{j}(d)$ as the capacity $d$ is implicitly given from the context.

Theorem 1 Let KP be a knapsack instance with optimal solution $x^{*}$ and let $K P_{\Delta p_{k}}$ be the instance where $p_{k}$ is substituted with $p_{k}^{\prime}=p_{k}+\Delta p_{k}$.

i) if $x_{k}^{*}=1$ then

$$
x^{*} \text { is optimal for } K P_{\Delta p_{k}} \Leftrightarrow \quad p_{k}^{\prime} \geq P
$$

ii) if $x_{k}^{*}=0$ then

$$
x^{*} \text { is optimal for } K P_{\Delta p_{k}} \Leftrightarrow 1 \leq p_{k}^{\prime} \leq P
$$

where

$$
P=z(K P \backslash\{k\})-z\left(K P\left[c-w_{k}\right] \backslash\{k\}\right) .
$$

Comment: The constraint $1 \leq p_{k}^{\prime}$ in (15) is only necessary to ensure that profits are positive as we assumed in the definition of (KP). If profits are allowed to be negative, then $p_{k}^{\prime}$ is downward unbounded.

Proof: The main idea in the proof is to find necessary and sufficient conditions for making the same choices as in the optimal solution in a dynamic programming recursion.

Since Recursion (4) does not demand any specific ordering of the item, we may swap item $k$ to the last position. Then the recursion says

$$
z\left(\mathrm{KP}_{\Delta p_{k}}\right)=\max \left\{z\left(\mathrm{KP}_{\Delta p_{k}} \backslash\{k\}\right), z\left(\mathrm{KP}_{\Delta p_{k}}\left[c-w_{k}\right] \backslash\{k\}\right)+p_{k}^{\prime}\right\}
$$

where the first term in the maximum expression corresponds to $x_{k}=0$ and the second term corresponds to $x_{k}=1$. Notice that if we choose the same term in (16) as in $x^{*}, x_{k}$ will correspond to $x_{k}^{*}$ and also the rest of the solution vector will be the same, since in order to find the solution vector we will backtrack from the same state in the dynamic programming recursion.

Since the only difference between $\mathrm{KP}$ and $\mathrm{KP}_{\Delta p_{k}}$ concerns element $k$, we have that

$$
\begin{aligned}
\mathrm{KP}_{\Delta p_{k}} \backslash\{k\} & =\mathrm{KP} \backslash\{k\}, \\
\mathrm{KP}_{\Delta p_{k}}\left[c-w_{k}\right] \backslash\{k\} & =\mathrm{KP}\left[c-w_{k}\right] \backslash\{k\} .
\end{aligned}
$$

Recursion (16) is hence equivalent to

$$
\begin{aligned}
z\left(\mathrm{KP}_{\Delta p_{k}}\right) & =\max \left\{z(\mathrm{KP} \backslash\{k\}), z\left(\mathrm{KP}\left[c-w_{k}\right] \backslash\{k\}\right)+p_{k}^{\prime}\right\} \\
& =\max \left\{z(\mathrm{KP} \backslash\{k\}), z(\mathrm{KP} \backslash\{k\})+p_{k}^{\prime}-P\right\} .
\end{aligned}
$$

Now, if $p_{k}^{\prime}-P \leq 0$ the first term in the maximum expression is the largest, while if $p_{k}^{\prime}-P \geq 0$ the second term is the largest. Since the first term corresponds to the case $x_{k}=0$, and the second term corresponds to the case $x_{k}=1$, the stated now follows directly. 
Theorem 2 Let KP be a knapsack instance with optimal solution $x^{*}$ and let $K P_{\Delta w_{k}}$ be the instance where $w_{k}$ is substituted with $w_{k}^{\prime}=w_{k}+\Delta w_{k}$.

i) if $x_{k}^{*}=1$ then

$$
x^{*} \text { is optimal for } K P_{\Delta w_{k}} \Leftrightarrow \quad c-W \leq w_{k}^{\prime} \leq w_{k}+r
$$

ii) if $x_{k}^{*}=0$ then

$$
x^{*} \text { is optimal for } K P_{\Delta w_{k}} \Leftrightarrow c-W \leq w_{k}^{\prime}
$$

where

$$
W=\max _{0 \leq d \leq c}\left\{d \mid z(K P[d] \backslash\{k\}) \leq z(K P)-p_{k}\right\}
$$

Comment: One could have expected that $w_{k}^{\prime}$ was downward unbounded in (21) similarly to Theorem 1. Indeed, decreasing $w_{k}^{\prime}$ will make it even more attractive to choose $x_{k}=1$, but if $w_{k}^{\prime}$ becomes too small, other items may fit into the knapsack and the optimal solution $x^{*}$ will change.

Proof: Since Recursion (4) does not demand any specific ordering of the items, we may swap item $k$ to the last position. Then the recursion says

$$
z\left(\mathrm{KP}_{\Delta w_{k}}\right)=\max \left\{z\left(\mathrm{KP}_{\Delta w_{k}} \backslash\{k\}\right), z\left(\mathrm{KP}_{\Delta w_{k}}\left[c-w_{k}^{\prime}\right] \backslash\{k\}\right)+p_{k}\right\}
$$

where the first term in the maximum expression corresponds to $x_{k}=0$ and the second term corresponds to $x_{k}=1$.

Notice that in the case $x_{k}^{*}=0$, as long as we choose the first term in (23), the whole solution vector $x^{*}$ will be unchanged, since we backtrack from the same state in the dynamic programming recursion.

The situation is different in the case $x_{k}^{*}=1$, since $z\left(\mathrm{KP}_{\Delta w_{k}}\left[c-w_{k}^{\prime}\right] \backslash\{k\}\right)$ refers to different states for each weight $w_{k}^{\prime}$. These states may lead to different solution vectors $x^{*}$ when backtracking through the dynamic programming table. Hence it is not sufficient to choose the second term in (23); we must also choose the exact same state $z\left(\mathrm{KP}_{\Delta w_{k}}\left[c-w_{k}\right] \backslash\{k\}\right)$. This can only be ensured if $z\left(\mathrm{KP}_{\Delta w_{k}}\left[c-w_{k}^{\prime}\right] \backslash\{k\}\right)=z\left(\mathrm{KP}_{\Delta w_{k}}\left[c-w_{k}\right] \backslash\{k\}\right)$, in which case we may choose state $z\left(\mathrm{KP}_{\Delta w_{k}}\left[c-w_{k}^{\prime}\right] \backslash\{k\}\right)$ instead of state $z\left(\mathrm{KP}_{\Delta w_{k}}\left[c-w_{k}\right] \backslash\{k\}\right)$.

Since the only difference between $\mathrm{KP}$ and $\mathrm{KP}_{\Delta w_{k}}$ concerns element $k$, we have that

$$
\begin{aligned}
\mathrm{KP}_{\Delta w_{k}} \backslash\{k\} & =\mathrm{KP} \backslash\{k\} \\
\mathrm{KP}_{\Delta w_{k}}\left[c-w_{k}^{\prime}\right] \backslash\{k\} & =\mathrm{KP}\left[c-w_{k}^{\prime}\right] \backslash\{k\} .
\end{aligned}
$$

This means that the Recursion (23) is equivalent to

$$
z\left(\mathrm{KP}_{\Delta w_{k}}\right)=\max \left\{z(\mathrm{KP} \backslash\{k\}), z\left(\mathrm{KP}\left[c-w_{k}^{\prime}\right] \backslash\{k\}\right)+p_{k}\right\} .
$$

In Case ii), where $x_{k}^{*}=0$, then the solution $x^{*}$ is unchanged under perturbation if and only if

$$
z(\mathrm{KP} \backslash\{k\}) \geq z\left(\operatorname{KP}\left[c-w_{k}^{\prime}\right] \backslash\{k\}\right)+p_{k},
$$


and since $x_{k}^{*}=0$ we have $z(\mathrm{KP} \backslash\{k\})=z(\mathrm{KP})$ and hence (27) is equivalent to

$$
z\left(\mathrm{KP}\left[c-w_{k}^{\prime}\right] \backslash\{k\}\right) \leq z(\mathrm{KP})-p_{k},
$$

which holds exactly for $c-w_{k}^{\prime} \leq W$.

In Case i), where $x_{k}^{*}=1$, then the solution $x^{*}$ is unchanged under perturbation if and only if

$$
z\left(\operatorname{KP}\left[c-w_{k}^{\prime}\right] \backslash\{k\}\right)=z\left(\operatorname{KP}\left[c-w_{k}\right] \backslash\{k\}\right) .
$$

We have that $z\left(\operatorname{KP}\left[c-w_{k}\right] \backslash\{k\}\right)=z(\mathrm{KP})-p_{k}$ so

$$
z\left(\mathrm{KP}\left[c-w_{k}^{\prime}\right] \backslash\{k\}\right)=z(\mathrm{KP})-p_{k},
$$

which holds exactly for $c-w_{k}^{\prime} \leq W \leq w_{k}+r$.

An illustrative example of Theorems 1 and 2 can be seen in Appendix A.

\subsection{Profit Tolerance Limits by Solving One KP}

Theorem 3 Let KP be a knapsack instance with optimal solution $x^{*}$ and let $K P_{\Delta p_{k}}$ be the instance where $p_{k}$ is substituted with $p_{k}^{\prime}=p_{k}+\Delta p_{k}$. The value of $P$ in Theorem 1 can be calculated as:

i) if $x_{k}^{*}=1$ then $P=z(K P \backslash\{k\})-z^{*}+p_{k}$

ii) if $x_{k}^{*}=0$ then $P=z^{*}-z\left(K P\left[c-w_{k}\right] \backslash\{k\}\right)$

Proof: We have $P=z(\operatorname{KP} \backslash\{k\})-z\left(\operatorname{KP}\left[c-w_{k}\right] \backslash\{k\}\right)$. If $x_{k}^{*}=1$ then $z\left(\operatorname{KP}\left[c-w_{k}\right] \backslash\{k\}\right)=$ $z^{*}-p_{k}$. If $x_{k}^{*}=0$ then $z(\mathrm{KP} \backslash\{k\})=z^{*}$.

The theorem shows that we only need to solve one knapsack problem to calculate $P$.

\subsection{Weight Tolerance Limits by Solving One KP}

Notice that for a given limit $z^{\prime}$ we have

$$
\max _{0 \leq d \leq c}\left\{d \mid z(\operatorname{KP}[d]) \leq z^{\prime}\right\}=\min _{0 \leq d \leq c+1}\left\{d \mid z(\operatorname{KP}[d]) \geq z^{\prime}+1\right\}-1=y\left(\operatorname{WKP}\left[z^{\prime}+1\right]\right)-1 .
$$

This means that we may calculate the tolerance limits in Theorem 2 as follows:

Theorem 4 Let $K P$ be a knapsack instance with optimal solution $x^{*}$ and let $K P_{\Delta w_{k}}$ be the instance where $w_{k}$ is substituted with $w_{k}^{\prime}=w_{k}+\Delta w_{k}$. The value of $W$ in Theorem 2 can be calculated as

$$
W=y\left(W K P\left[z^{*}-p_{k}+1\right] \backslash\{k\}\right)-1 .
$$

Proof: Since $z(\mathrm{KP})=z^{*}$ it follows from Equation (31) that

$$
W=\max _{0 \leq d \leq c}\left\{d \mid z(\operatorname{KP}[d] \backslash\{k\}) \leq z^{*}-p_{k}\right\}=y\left(\operatorname{WKP}\left[z^{*}-p_{k}+1\right] \backslash\{k\}\right)-1 .
$$




\subsection{Algorithm to Determine Tolerance Intervals}

Theorems 1, 2, 3 and 4 provide us with the following tolerance limits for a given item $k$.

Algorithm 1 Assume that $x^{*}$ is an optimal solution to KP with solution value $z^{*}$ and residual capacity $r$. The tolerance limits for items $k=1,2, \ldots, n$ can then be calculated as:

$$
\begin{aligned}
& \text { if } x_{k}^{*}=1 \text { then } \begin{cases}\alpha_{p_{k}}=z(K P[c] \backslash\{k\})-z^{*}+p_{k} & \beta_{p_{k}}=\infty \\
\alpha_{w_{k}}=c-y\left(W K P\left[z^{*}-p_{k}+1\right] \backslash\{k\}\right)+1 & \beta_{w_{k}}=w_{k}+r\end{cases} \\
& \text { if } x_{k}^{*}=0 \text { then } \begin{cases}\alpha_{p_{k}}=0 & \beta_{p_{k}}=z^{*}-z\left(K P\left[c-w_{k}\right] \backslash\{k\}\right) \\
\alpha_{w_{k}}=c-y\left(W K P\left[z^{*}-p_{k}+1\right] \backslash\{k\}\right)+1 & \beta_{w_{k}}=\infty\end{cases}
\end{aligned}
$$

If $\alpha_{p_{k}}<0$ we set $\alpha_{p_{k}}=0$, and if $\alpha_{w_{k}}<0$ we set $\alpha_{w_{k}}=0$ to ensure nonnegative coefficients.

A possible implementation of the above algorithm is for each $k=1,2, \ldots, n$ to remove item $k$ from the problem and solve the remaining problem by use of Recursion (4) in time $O(n c)$. Finding all profit tolerance limits can hence be done in $O\left(n^{2} c\right)$. A similar approach is used for the weight tolerance limits.

\section{Faster Tolerance Analysis}

In the previous section we saw that the tolerance limits can be found in $O(n c)$ time for each item. This is better than the naïve algorithm presented in Section 3.

In this section we show how the time complexity can be further decreased by reusing parts of the dynamic programming table, leading to an amortized running time of $O(c \log n)$ per item. Moreover, we show how tolerance limits can be found by solving $n$ ordinary $0-1$ knapsack problems. Finally we show how approximate tolerance limits can be found in polynomial time by use of various upper bounds.

\subsection{Overlapping Subproblems}

If we use dynamic programming to find tolerance intervals for all items, large parts of the dynamic programming table will be the same. Indeed, our solution approach only demands that one item $k$ is removed from the problem.

This can be exploited in a tree structure as illustrated in Fig. 1. The considered instance has 8 items, and hence we need to run dynamic programming where each of the 8 items in turn has been removed. This is shown in the last row of the figure, where each set on the form $\{1,2,3,4,5,6,7\}$ shows the order in which the items are considered in the dynamic programming Recursion (4). Higher up in the tree, we show the items which have been considered in Recursion (4). The item numbers in bold are the new items added from the above level.

In each row $i$ of the tree we add $n / 2^{i}$ items to each of $2^{i}$ subproblems, and there are $\left\lceil\log _{2} n\right\rceil$ rows. Addition of one item by use of Recursion (4) takes $O(c)$ computation. This means that each row can be evaluated in $O(n c)$. Hence, the overall running time is $O(n c \log n)$, since we have $O(\log n)$ rows. The amortized time complexity for each item now becomes $O(c \log n)$. 


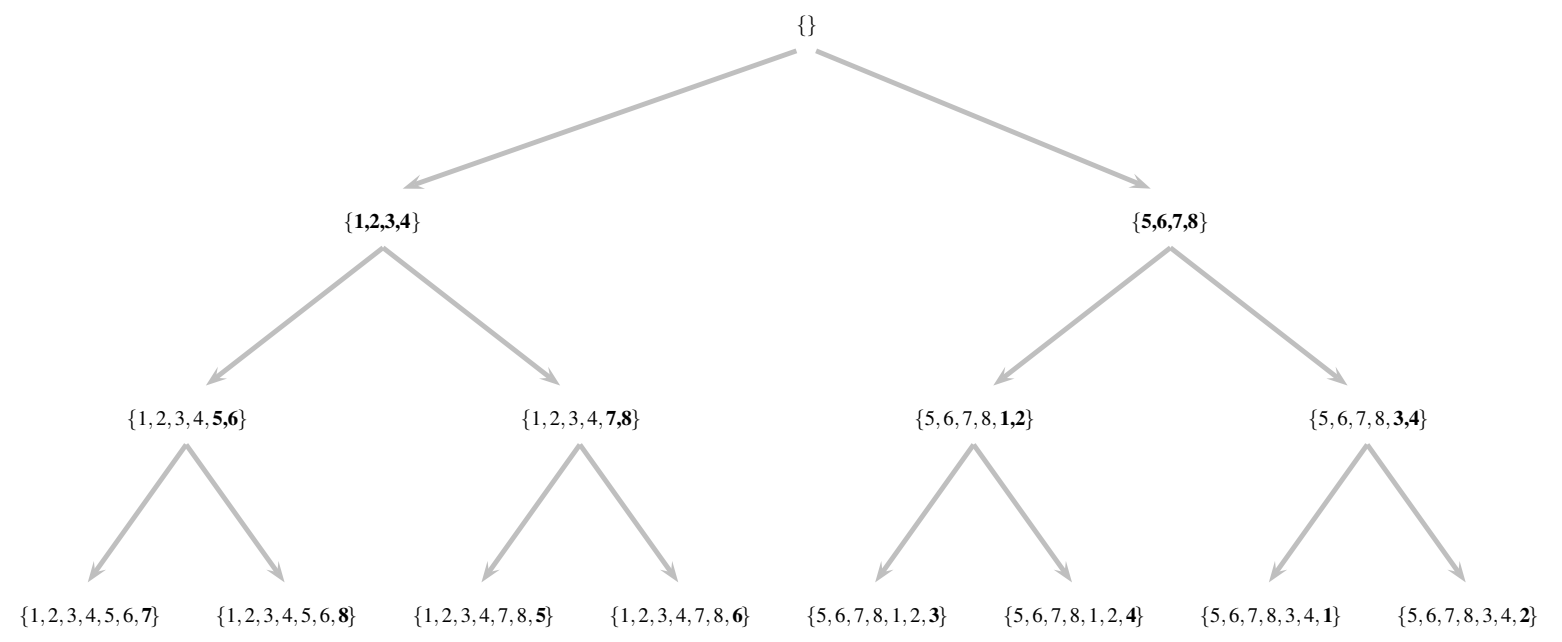

Figure 1: Tree structure which makes use of overlapping subproblems to solve $n$ related dynamic programming problems. In each set, the item numbers in bold refers to new items.

\subsection{Efficient Algorithms for Subproblems}

Although the proof of Theorems 1 and 2 made use of dynamic programming, we are not limited to use this paradigm. Indeed we just need to solve the problems

$$
z(\operatorname{KP}[c] \backslash\{k\}), \quad z\left(\operatorname{KP}\left[c-w_{k}\right] \backslash\{k\}\right), \quad y\left(\mathrm{WKP}\left[z^{*}-p_{k}\right] \backslash\{k\}\right) .
$$

We can use any state-of-the-art algorithm for solving these subproblems, e.g., the algorithms presented in $[15,19]$. Although these algorithms have a worst-case complexity $O(n c)$, they are much faster in practice.

\subsection{Upper Bounds}

Instead of solving Subproblems (32) to optimality we may use any upper bound on the solution value to obtain valid (but not necessarily optimal) tolerance limits. Let $\mathscr{U}(\mathrm{KP})$ be an upper bound on $z(\mathrm{KP})$ and let $\mathscr{L}(\mathrm{WKP})$ be a lower bound on $y(\mathrm{WKP})$. Then we have

Algorithm 2 Approximate tolerance limits can be found as

$$
\begin{aligned}
& \text { if } x_{k}^{*}=1 \text { then } \begin{cases}\alpha_{p_{k}}=\mathscr{U}(K P[c] \backslash\{k\})-z^{*}+p_{k} & \beta_{p_{k}}=\infty \\
\alpha_{w_{k}}=c-\mathscr{L}\left(W K P\left[z^{*}-p_{k}+1\right] \backslash\{k\}\right)+1 & \beta_{w_{k}}=w_{k}+r\end{cases} \\
& \text { if } x_{k}^{*}=0 \text { then } \begin{cases}\alpha_{p_{k}}=0 & \beta_{p_{k}}=z^{*}-\mathscr{U}\left(K P\left[c-w_{k}\right] \backslash\{k\}\right) \\
\alpha_{w_{k}}=c-\mathscr{L}\left(W K P\left[z^{*}-p_{k}+1\right] \backslash\{k\}\right)+1 & \beta_{w_{k}}=\infty\end{cases}
\end{aligned}
$$

If the upper/lower bounds are sufficiently loose the above equations may return $\alpha_{p_{k}}>p_{k}$ or $\beta_{p_{k}}<p_{k}$. In the first case we set $\alpha_{p_{k}}=p_{k}$, and in the second case we set $\beta_{p_{k}}=w_{k}$. 
Proof: To see the correctness of the above, observe that if $\mathscr{U}(\mathrm{KP}[c] \backslash\{k\}) \geq z(\operatorname{KP}[c] \backslash\{k\})$ then

$$
\mathscr{U}(\mathrm{KP}[c] \backslash\{k\})-z^{*}+p_{k} \geq z(\operatorname{KP}[c] \backslash\{k\})-z^{*}+p_{k}=\alpha_{p_{k}}
$$

Moreover, if $\mathscr{U}\left(\operatorname{KP}\left[c-w_{k}\right] \backslash\{k\}\right) \geq z\left(\operatorname{KP}\left[c-w_{k}\right] \backslash\{k\}\right)$ then

$$
z^{*}-\mathscr{U}\left(\operatorname{KP}\left[c-w_{k}\right] \backslash\{k\}\right) \leq z^{*}-z\left(\operatorname{KP}\left[c-w_{k}\right] \backslash\{k\}\right)=\beta_{p_{k}} .
$$

For the weight tolerance intervals we have that if $\mathscr{L}\left(\mathrm{WKP}\left[z^{*}-p_{k}+1\right] \backslash\{k\}\right) \leq y\left(\mathrm{WKP}\left[z^{*}-\right.\right.$ $\left.\left.p_{k}+1\right] \backslash\{k\}\right)$ then

$$
c-\mathscr{L}\left(\mathrm{WKP}\left[z^{*}-p_{k}+1\right] \backslash\{k\}\right)+1 \geq c-y\left(\mathrm{WKP}\left[z^{*}-p_{k}+1\right] \backslash\{k\}\right)+1=\alpha_{w_{k}} .
$$

Together with Algorithm 1 we get the stated.

Proposition 1 If we use the Dantzig Bound in Algorithm 2 we get approximate tolerance limits in amortized time $O(\log n)$ per item.

Proof: We first sort the items according to nonincreasing profit-to-weight ratio in time $O(n \log n)$, and then we calculate the accumulated weight sums $\bar{w}_{j}=\sum_{i=1}^{j} w_{i}$ and profit sums $\bar{p}_{j}=\sum_{i=1}^{j} p_{i}$ in overall linear time. For each item $k$ we can then calculate a bound on $\mathrm{KP} \backslash\{k\}$ by using binary search to find the split item $s$ as the item satisfying $\bar{w}_{s-1} \leq c<\bar{w}_{s}$, and then calculate the Dantzig Bound as $\bar{p}_{s-1}+\left(c-\bar{w}_{s-1}\right) p_{s} / w_{s}$.

Proposition 2 If we use the Dembo-Hammer upper bound [5] in Algorithm 2 we may find approximate tolerance limits in time $O(1)$ per item.

The Dembo-Hammer upper bound is defined as follows: Let $z_{L P}^{*}$ be the Dantzig upper bound given by (2), and $p_{s}$ and $w_{s}$ be the profit and weight of the corresponding split item $s$. For any perturbation $\Delta w$, the Dembo-Hammer bound is then

$$
\mathscr{U}(\mathrm{KP}[c+\Delta w])=z_{L P}^{*}+\Delta w \frac{p_{s}}{w_{s}}
$$

Notice, that the same split item $s$ is used for all capacities, and all sub-instances of KP, making it possible to calculate the bound in $O(1)$ once $s$ has been found. This leads to the following algorithm

Algorithm 3 Let $z_{L P}^{*}, p_{s}$ and $w_{s}$ be defined as above in (33). The tolerance limits are then given as

$$
\begin{aligned}
& \text { if } x_{k}^{*}=1 \text { then } \begin{cases}\alpha_{p_{k}}=p_{k}+z_{L P}^{*}-z^{*} & \beta_{p_{k}}=\infty \\
\alpha_{w_{k}}=c+1-z_{L P}^{w *}-\left(p_{k}-1\right) w_{s} / p_{s} & \beta_{w_{k}}=w_{k}+c-z_{L P}^{w *}-\left(p_{k}\right) w_{s} / p_{s}\end{cases} \\
& \text { if } x_{k}^{*}=0 \text { then } \begin{cases}\alpha_{p_{k}}=0 & \beta_{p_{k}}=z^{*}-z_{L P}^{*}-w_{k} p_{s} / w_{s} \\
\alpha_{w_{k}}=c+1-z_{L P}^{w *}-\left(p_{k}-1\right) w_{s} / p_{s} & \beta_{w_{k}}=\infty\end{cases}
\end{aligned}
$$


Proof: We have

$$
\begin{aligned}
\mathscr{U}(\mathrm{KP}[c] \backslash\{k\}) & =z_{L P}^{*}, \\
\mathscr{U}\left(\mathrm{KP}\left[c-w_{k}\right] \backslash\{k\}\right) & =z_{L P}^{*}-w_{k} p_{s} / w_{s}, \\
\mathscr{L}\left(\mathrm{WKP}\left[z^{*}-p_{k}+1\right] \backslash\{k\}\right) & =z_{L P}^{w *}-\left(p_{k}-1\right) w_{s} / p_{s}, \\
\mathscr{L}\left(\mathrm{WKP}\left[z^{*}-p_{k}\right] \backslash\{k\}\right) & =z_{L P}^{w *}-\left(p_{k}\right) w_{s} / p_{s} .
\end{aligned}
$$

Inserting the bounds in Algorithm 2 we get the stated.

Since the Dantzig bound is tighter than the Dembo-Hammer bound we get tighter tolerance limits by using the first-mentioned bound. The time-complexity of the Dantzig bound is, however, larger.

\section{Experimental Results}

The object of this section is to compare the approximate method proposed by Hifi et al. [11] with the new exact method proposed in this paper with respect to quality and computing times. In addition we discuss the tradeoffs between the approximate method compared to the exact method.

The code used for the tests in this paper has been implemented in $\mathrm{C} / \mathrm{C}++$ and run using $g c c$ on a Intel Xeon 5365 QuadCore running at $2.66 \mathrm{GHz}$. The reported running times are for a single core.

In the sequel we will use the following naming convention: Let $\mathrm{Na}$ ïve be the naïve algorithm using Equations (9)-(10) and (12)-(13). Let ExactDP be the algorithm based on dynamic programming described in Section 5.1 which makes use of overlapping subproblems. Let ExactKP be the algorithm based on solving a number of knapsack and weight knapsack problems as described in Algorithm 1. Let ApproxLP be Algorithm 2 using the Dantzig upper bound for calculating the tolerance limits, and let ApproxDH be Algorithm 2 using the Dembo-Hammer upper bound for calculating the tolerance limits. In both Nä̈ve and Exact KP we use the highly efficient Combo algorithm [15] for solving the individual knapsack problems to optimality.

\subsection{Instance from the Literature}

In this section we compare ExactDP with ApproxLP on the twenty item example presented in Hifi et al. [11], page 257 with capacity $c$ equal to 420 . The results have been slightly corrected as the original table contained a typo [9].

Table 3 shows the correct data for this example. The optimal solution value $z^{*}=709$. The profit tolerance limits in Columns 5 and 6 are the approximate limits computed by ApproxLP described in Section 5.3 while Columns 7 and 8 show the exact intervals as computed by ExactDP. Columns 9 and 10 report the deviation between the two methods. A 100\% deviation corresponds to the case, where ApproxLP found a trivial tolerance limit equal to the original profit, while ExactDP found a nontrivial tolerance limit. 
Table 3: Example proposed by Hifi et al. [11] showing the profit and weight tolerance limits. The knapsack has capacity $c=420$. For the profits, Columns 5 and 6 show the limits computed with ApproxLP. Columns 7 and 8 show the exact limits as computed by ExactDP. Columns 9 and 10 report the deviation in percent as $\operatorname{dev}_{\alpha}=100\left(\alpha_{p_{k}}-\alpha_{p_{k}}^{\prime}\right) /\left(p_{k}-\alpha_{p_{k}}^{\prime}\right)$ and an equivalent equation for $\operatorname{dev}_{\beta}$. For the weights, Columns 11 to 16 show the respective values.

\begin{tabular}{|c|c|c|c|c|c|c|c|c|c|c|c|c|c|c|c|}
\hline \multirow{3}{*}{$\frac{\text { Item }}{k}$} & \multirow{2}{*}{\multicolumn{2}{|c|}{ Parameters }} & \multirow{3}{*}{$\frac{\text { Solution }}{x_{k}^{*}}$} & \multicolumn{6}{|c|}{ Profit tolerance limits } & \multicolumn{6}{|c|}{ Weight tolerance limits } \\
\hline & & & & \multicolumn{2}{|c|}{ ApproxLP } & \multicolumn{2}{|c|}{ ExactDP } & \multicolumn{2}{|c|}{ Deviation in \% } & \multicolumn{2}{|c|}{ ApproxLP } & \multicolumn{2}{|c|}{ ExactDP } & \multicolumn{2}{|c|}{ Deviation in $\%$} \\
\hline & $p_{k}$ & $w_{k}$ & & $\alpha_{p_{k}}$ & $\beta_{p_{k}}$ & $\alpha_{p_{k}}^{\prime}$ & $\beta_{p_{k}}^{\prime}$ & $\operatorname{dev}_{\alpha}$ & $\operatorname{dev}_{\beta}$ & $\alpha_{w_{k}}$ & $\beta_{w_{k}}$ & $\alpha_{w_{k}}^{\prime}$ & $\beta_{w_{k}}^{\prime}$ & $\operatorname{dev}_{\alpha}$ & $\operatorname{dev}_{\beta}$ \\
\hline 1 & 80 & 4 & 1 & 17 & $\infty$ & 3 & $\infty$ & 18.2 & 0.0 & 4 & 16 & 2 & 16 & 100.0 & 0.0 \\
\hline 2 & 28 & 3 & 1 & 16 & $\infty$ & 3 & $\infty$ & 52.0 & 0.0 & 3 & 15 & 1 & 15 & 100.0 & 0.0 \\
\hline 3 & 54 & 15 & 1 & 28 & $\infty$ & 6 & $\infty$ & 45.8 & 0.0 & 15 & 27 & 13 & 27 & 100.0 & 0.0 \\
\hline 4 & 81 & 25 & 1 & 37 & $\infty$ & 33 & $\infty$ & 8.3 & 0.0 & 25 & 37 & 23 & 37 & 100.0 & 0.0 \\
\hline 5 & 31 & 12 & 1 & 25 & $\infty$ & 3 & $\infty$ & 78.6 & 0.0 & 12 & 24 & 10 & 24 & 100.0 & 0.0 \\
\hline 6 & 30 & 17 & 1 & 30 & $\infty$ & 6 & $\infty$ & 100.0 & 0.0 & 17 & 29 & 10 & 29 & 100.0 & 0.0 \\
\hline 7 & 39 & 24 & 1 & 36 & $\infty$ & 33 & $\infty$ & 50.0 & 0.0 & 24 & 36 & 22 & 36 & 100.0 & 0.0 \\
\hline 8 & 41 & 27 & 1 & 39 & $\infty$ & 33 & $\infty$ & 75.0 & 0.0 & 27 & 39 & 25 & 39 & 100.0 & 0.0 \\
\hline 9 & 68 & 51 & 1 & 61 & $\infty$ & 44 & $\infty$ & 70.0 & 0.0 & 51 & 63 & 49 & 63 & 100.0 & 0.0 \\
\hline 10 & 83 & 65 & 1 & 74 & $\infty$ & 74 & $\infty$ & 0.0 & 0.0 & 65 & 77 & 63 & 77 & 100.0 & 0.0 \\
\hline 11 & 33 & 30 & 1 & 33 & $\infty$ & 33 & $\infty$ & 0.0 & 0.0 & 30 & 42 & 28 & 42 & 100.0 & 0.0 \\
\hline 12 & 100 & 91 & 1 & 92 & $\infty$ & 80 & $\infty$ & 60.0 & 0.0 & 91 & 103 & 89 & 103 & 100.0 & 0.0 \\
\hline 13 & 74 & 76 & 0 & 0 & 74 & 0 & 74 & 0.0 & 0.0 & 76 & $\infty$ & 74 & $\infty$ & 100.0 & 0.0 \\
\hline 14 & 41 & 44 & 1 & 41 & $\infty$ & 41 & $\infty$ & 0.0 & 0.0 & 44 & 56 & 35 & 56 & 100.0 & 0.0 \\
\hline 15 & 47 & 70 & 0 & 0 & 57 & 0 & 67 & 0.0 & 50.0 & 61 & $\infty$ & 57 & $\infty$ & 30.8 & 0.0 \\
\hline 16 & 38 & 69 & 0 & 0 & 56 & 0 & 67 & 0.0 & 37.9 & 52 & $\infty$ & 43 & $\infty$ & 34.6 & 0.0 \\
\hline 17 & 32 & 86 & 0 & 0 & 74 & 0 & 74 & 0.0 & 0.0 & 46 & $\infty$ & 30 & $\infty$ & 28.6 & 0.0 \\
\hline 18 & 16 & 62 & 0 & 0 & 48 & 0 & 67 & 0.0 & 37.3 & 30 & $\infty$ & 13 & $\infty$ & 34.7 & 0.0 \\
\hline 19 & 6 & 29 & 0 & 0 & 15 & 0 & 30 & 0.0 & 62.5 & 20 & $\infty$ & 13 & $\infty$ & 43.8 & 0.0 \\
\hline 20 & 8 & 40 & 0 & 0 & 26 & 0 & 33 & 0.0 & 28.0 & 22 & $\infty$ & 13 & $\infty$ & 33.3 & 0.0 \\
\hline
\end{tabular}

As for the weight tolerance limits, the respective values are reported in Columns 11 to 16. It is interesting to see that the lower limit using ApproxLP very frequently just returns the original weight $w_{k}$. For the upper limits, both ApproxLP and ExactDP return the same value, since both equations only depend on the residual capacity $r$.

\subsection{Large Instances}

In this section we compare the various algorithms on large instances to examine the tradeoffs between quality and computation time. We consider four categories of randomly generated knapsack instances, which have been constructed to reflect special properties that may influence the solution process. The four instance groups are: uncorrelated, weakly correlated, strongly correlated and subset sum instances [16]. In all four groups the weights are randomly chosen as integers from a discrete uniform distribution $[1, R]$. The profits are then expressed as a function of the weights, yielding the specific properties of each group: Uncorrelated instances; The parameters $p_{j}$ and $w_{j}$ are randomly chosen as integers in $[1, R]$. Weakly correlated instances; Each weight $w_{j}$ is chosen as a random integer in $[1, R]$ and each profit $p_{j}$ is chosen as a random integer in $\left[w_{j}-R / 10, w_{j}+R / 10\right]$ such that $p_{j} \geq 1$. Strongly correlated instances; The weights $w_{j}$ are distributed as integers in $[1, R]$ and $p_{j}=w_{j}+R / 10$. Subset sum instances; The weights are randomly distributed integers in $[1, R]$ and $p_{j}=w_{j}$. Notice, that the profits and weights are 
Table 4: Total running times in milliseconds on a Dell Optiflex 9020 with i7-4790 Processor (3.6 GHz). The reported times are for finding all tolerance limits, include solution of the original instance. Average values of $N=10$ instances.

\begin{tabular}{|l|r|rrrr|}
\hline Algorithm & \multicolumn{1}{|c|}{$n$} & uncorrelated & weakly corr. & str. corr. & subset-sum \\
\hline Nä̈ve & 5 & 34 & 13 & 11 & 9 \\
& 10 & 74 & 30 & 15 & 7 \\
& 20 & 186 & 72 & 46 & 19 \\
& 50 & 662 & 323 & 4656 & 38 \\
& 100 & 1791 & 976 & 72383 & 70 \\
& 200 & 6390 & 3251 & 212511 & 155 \\
& 500 & 32919 & 26010 & 3774158 & 342 \\
& 1000 & 165496 & 88267 & 19899575 & 634 \\
\hline ExactKP & 5 & 0 & 0 & 0 & 0 \\
& 10 & 0 & 0 & 0 & 0 \\
& 20 & 0 & 0 & 1 & 19 \\
& 50 & 1 & 2 & 30 & 43 \\
& 100 & 2 & 4 & 75 & 72 \\
& 200 & 7 & 13 & 153 & 124 \\
& 500 & 26 & 88 & 613 & 311 \\
& 1000 & 108 & 275 & 1074 & 642 \\
\hline ApproxLP & 5 & 0 & 0 & 0 & 0 \\
& 10 & 0 & 0 & 0 & 0 \\
& 20 & 0 & 0 & 0 & 0 \\
& 50 & 0 & 0 & 2 & 1 \\
& 100 & 0 & 1 & 2 & 1 \\
& 200 & 1 & 1 & 0 & 2 \\
& 500 & 4 & 4 & 6 & 1 \\
& 1000 & 14 & 16 & 15 & 9 \\
\hline ApproxDH & 5 & 0 & 0 & 0 & 0 \\
& 10 & 0 & 0 & 0 & 0 \\
& 20 & 0 & 0 & 0 & 0 \\
& 50 & 0 & 0 & 1 & 0 \\
& 100 & 0 & 0 & 1 & 0 \\
& 200 & 0 & 0 & 0 & 1 \\
& 500 & 0 & 0 & 0 & 1 \\
& 1000 & 0 & 0 & 0 & 0 \\
\hline
\end{tabular}

perturbed independently, hence the instances are not subset sum instances after perturbation.

To generate knapsack instances with the four properties, we use a knapsack generator described in Pisinger [20]. For each group we generate instances, whose sizes $n$ vary between 5 and 1,000. For each size $n$ we generate $N=10$ instances. The capacity is $c=\sum w_{j} / 2$.

We evaluate the performance of the presented algorithms on instances with relatively large profits and weights, using $R=10,000$. For small values of $R$ the tolerance limits frequently become trivial.

Preliminary experiments showed that the dynamic programming algorithm ExactDP with complexity $O(n c \log n)$ was not competitive with ExactKP in time $O\left(n^{2} c\right)$, and ran out of space for large values of $c$. Hence we do not report running times for ExactDP.

Table 4 first compares the running times of the various approaches. The running times are for finding all tolerance limits, and includes the solution of the original instance. It is seen that the naïve approach Nä̈ve is very time-consuming using several minutes for large-sized uncorrelated instances, and growing to several hours for large-sized strongly correlated instances. On the contrary, the improved exact algorithm ExactKP finds all the tolerance limits in a less than a second. The approximate algorithm ApproxLP using the Dantzig upper bound is very 
Table 5: Average value of $S_{p_{k}}$ (left) and average value of $S_{w_{k}}$ (right).

\begin{tabular}{|c|c|c|c|c|c|c|c|c|c|}
\hline \multirow[b]{2}{*}{ Algorithm } & \multirow[b]{2}{*}{$n$} & \multicolumn{4}{|c|}{ avg. $S_{p_{k}}$} & \multicolumn{4}{|c|}{$\operatorname{avg} . S_{w_{k}}$} \\
\hline & & uncorrelated & weakly corr. & str. corr. & subset-sum & uncorrelated & weakly corr. & str. corr. & subset-sum \\
\hline \multirow[t]{8}{*}{ ExactKP } & 5 & 3937.6 & 1133.9 & 1287.6 & 1115.3 & 2991.1 & 1361.7 & 850.2 & 751.0 \\
\hline & 10 & 2885.1 & 696.2 & 427.6 & 55.4 & 2418.5 & 640.6 & 255.2 & 43.0 \\
\hline & 20 & 3238.5 & 612.7 & 321.4 & 0.0 & 2044.7 & 413.5 & 73.9 & 0.0 \\
\hline & 50 & 3394.2 & 493.5 & 210.7 & 0.0 & 1746.0 & 282.4 & 28.1 & 0.0 \\
\hline & 100 & 3175.8 & 492.1 & 349.6 & 0.0 & 1407.0 & 254.3 & 23.7 & 0.0 \\
\hline & 200 & 3176.6 & 512.1 & 607.5 & 0.0 & 1285.8 & 254.3 & 98.9 & 0.0 \\
\hline & 500 & 3107.4 & 501.1 & 216.8 & 0.0 & 1248.7 & 247.2 & 8.1 & 0.0 \\
\hline & 1000 & 3104.4 & 506.1 & 437.0 & 0.0 & 1210.1 & 252.7 & 38.8 & 0.0 \\
\hline \multirow[t]{8}{*}{ ApproxLP } & 5 & 1710.3 & 6.5 & 16.1 & 0.0 & 1797.7 & 528.2 & 242.1 & 169.6 \\
\hline & 10 & 1529.6 & 117.6 & 40.3 & 0.0 & 1583.8 & 175.2 & 82.6 & 7.7 \\
\hline & 20 & 2016.4 & 152.4 & 59.4 & 0.0 & 1439.7 & 177.8 & 17.5 & 0.0 \\
\hline & 50 & 2603.2 & 270.0 & 53.2 & 0.0 & 1116.8 & 157.6 & 5.0 & 0.0 \\
\hline & 100 & 2711.3 & 353.7 & 98.9 & 0.0 & 1118.2 & 179.7 & 4.1 & 0.0 \\
\hline & 200 & 2898.8 & 410.2 & 205.6 & 0.0 & 1137.7 & 202.4 & 18.4 & 0.0 \\
\hline & 500 & 2979.6 & 460.5 & 51.3 & 0.0 & 1173.1 & 226.0 & 1.0 & 0.0 \\
\hline & 1000 & 3026.4 & 476.9 & 129.1 & 0.0 & 1169.3 & 235.7 & 5.9 & 0.0 \\
\hline \multirow[t]{8}{*}{ ApproxDH } & 5 & 177.6 & 5.9 & 0.0 & 0.0 & 288.1 & 95.2 & 4.1 & 24.4 \\
\hline & 10 & 271.5 & 67.1 & 3.5 & 0.0 & 353.4 & 81.5 & 3.0 & 0.0 \\
\hline & 20 & 690.4 & 80.3 & 2.3 & 0.0 & 806.9 & 90.7 & 2.0 & 0.0 \\
\hline & 50 & 749.4 & 128.1 & 3.2 & 0.0 & 982.7 & 132.2 & 2.8 & 0.0 \\
\hline & 100 & 757.2 & 156.4 & 4.0 & 0.0 & 976.0 & 157.9 & 3.6 & 0.0 \\
\hline & 200 & 845.9 & 189.5 & 18.3 & 0.0 & 1073.3 & 190.6 & 16.1 & 0.0 \\
\hline & 500 & 865.2 & 220.6 & 1.1 & 0.0 & 1137.1 & 220.8 & 1.0 & 0.0 \\
\hline & 1000 & 868.5 & 232.3 & 6.6 & 0.0 & 1155.5 & 232.5 & 5.8 & 0.0 \\
\hline
\end{tabular}

Table 6: Number of items where $S_{p_{k}}>0$ (left) and number of items where $S_{w_{k}}>0$ (right).

\begin{tabular}{|c|c|c|c|c|c|c|c|c|c|}
\hline \multirow{2}{*}{ Algorithm } & \multirow[b]{2}{*}{$n$} & \multicolumn{4}{|c|}{$S_{p_{k}}>0$} & \multicolumn{4}{|c|}{$S_{w_{k}}>0$} \\
\hline & & uncorrelated & weakly corr. & str. corr. & subset-sum & uncorrelated & weakly corr. & str. corr. & subset-sum \\
\hline \multirow[t]{8}{*}{ ExactKP } & 5 & 5 & 5 & 5 & 5 & 5 & 5 & 5 & 5 \\
\hline & 10 & 10 & 10 & 10 & 10 & 10 & 10 & 10 & 10 \\
\hline & 20 & 20 & 20 & 19 & 0 & 20 & 20 & 20 & 0 \\
\hline & 50 & 50 & 50 & 22 & 0 & 50 & 50 & 8 & 0 \\
\hline & 100 & 100 & 100 & 47 & 0 & 100 & 100 & 14 & 0 \\
\hline & 200 & 200 & 200 & 133 & 0 & 200 & 200 & 60 & 0 \\
\hline & 500 & 500 & 499 & 117 & 0 & 500 & 500 & 9 & 0 \\
\hline & 1000 & 1000 & 999 & 444 & 0 & 1000 & 950 & 65 & 0 \\
\hline \multirow[t]{8}{*}{ ApproxLP } & 5 & 3 & 0 & 0 & 0 & 4 & 3 & 3 & 2 \\
\hline & 10 & 6 & 4 & 2 & 0 & 8 & 7 & 6 & 5 \\
\hline & 20 & 16 & 10 & 5 & 0 & 18 & 16 & 11 & 0 \\
\hline & 50 & 44 & 34 & 10 & 0 & 47 & 42 & 5 & 0 \\
\hline & 100 & 91 & 83 & 35 & 0 & 96 & 91 & 10 & 0 \\
\hline & 200 & 188 & 180 & 121 & 0 & 194 & 189 & 55 & 0 \\
\hline & 500 & 485 & 475 & 108 & 0 & 491 & 462 & 8 & 0 \\
\hline & 1000 & 977 & 974 & 437 & 0 & 989 & 886 & 62 & 0 \\
\hline \multirow[t]{8}{*}{ ApproxDH } & 5 & 0 & 0 & 0 & 0 & 0 & 0 & 0 & 0 \\
\hline & 10 & 1 & 2 & 0 & 0 & 1 & 2 & 0 & 0 \\
\hline & 20 & 6 & 5 & 0 & 0 & 6 & 5 & 0 & 0 \\
\hline & 50 & 15 & 16 & 1 & 0 & 16 & 17 & 1 & 0 \\
\hline & 100 & 32 & 39 & 3 & 0 & 33 & 40 & 3 & 0 \\
\hline & 200 & 69 & 87 & 26 & 0 & 69 & 87 & 26 & 0 \\
\hline & 500 & 178 & 232 & 7 & 0 & 178 & 232 & 7 & 0 \\
\hline & 1000 & 360 & 483 & 62 & 0 & 360 & 483 & 62 & 0 \\
\hline
\end{tabular}


fast, using no more than 0.02 seconds for all large-scaled instances. The fastest algorithm is the ApproxDH which only spends a few milliseconds to find the tolerance limits.

The size of a tolerance interval for $p_{k}$ and $w_{k}$ is $S_{p_{k}}=\left(\beta_{p_{k}}-\alpha_{p_{k}}\right)$ and $S_{w_{k}}=\left(\beta_{w_{k}}-\alpha_{w_{k}}\right)$, respectively. Table 5 reports the average value of $S_{p_{k}}$ and $S_{w_{k}}$. It is seen that the tolerance limits decrease for increasing correlation. For the most correlated instances, the subset-sum instances, the tolerance limits are zero except for very small instances. The range of the tolerance intervals for the weights are significantly smaller then the tolerance intervals for the profits. For the strongly correlated instances, the average lower tolerance limits are less then $2 \%$ on average.

Comparing the values of the tolerance limits in Table 5, it is seen that ApproxLP using the Dantzig upper bound is close to the exact tolerance limits for large-sized uncorrelated, weakly correlated and subset-sum instances. However, for small-sized instances the use of the Dantzig upper bound significantly decreases the tolerance limits. The ApproxDH algorithm using the Dembo-Hammer upper bound finds considerably smaller tolerance limits than the previous approaches for the profits, and hence is hardly of any use. However for the weights ApproxDH finds comparable tolerance limits.

Finally, Table 6 reports the number of items with non-zero tolerance limits. It is seen that for the uncorrelated and weakly correlated instances nearly all items have a non-trivial exact tolerance limit for the profits and the weights. For the strongly correlated instance around half of the items have a non-zero tolerance limit for the profits, and considerably fewer for the weights. For the subset-sum instance, non-trivial tolerance limits are found only for small instances. This may be due to the fact that for larger instances numerous solutions exist with objective value equal to the capacity $c$, and hence a small increase in the profit or weight of an item will change the solution.

\section{Conclusion}

Tolerance analysis makes it possible to test the robustness of a model, and to fix model input that has no effect on the output. Knapsack problems frequently occur as subproblems in more complex combinatorial algorithms, e.g., as pricing problem (Vanderbeck [23]) or for cut separation (Balas [2], Fukasawa and Goycoolea [6]). Knowing the tolerance limits can perhaps be used to derive tighter cuts, and it can save time in solving pricing problems when the parameters only have been changed within the tolerance limits.

We have presented an algorithm for finding the exact tolerance limits in amortized time $O(c \log n)$ for each item. Moreover we have showed that the tolerance limits can be found by solving a single knapsack problem, making it possible to use all current knapsack solvers and upper bound algorithms for calculating the limits.

Notice, that although we used dynamic programming (or actually a recursive formulation) to prove the theorems, the found tolerance limits are independent of dynamic programming. This means that the theorems, with a few modifications, also hold if the profits and weights are rational numbers.

The computation experiments show that the exact algorithm ExactKP is performing very well, making it possible to find all tolerance limits in roughly one second for large-sized in- 
stances. If faster solution times are needed, one may use the ApproxLP algorithm for larger instances (say, $n>100$ ) and ExactKP for the smaller instances. This combination results in very fast running times, and a good quality of the tolerance limits.

Future work should focus on how the presented results can be generalized to other problems that can be solved in pseudo-polynomial time. It should be possible to use similar arguments from dynamic programming to analyze the tolerance limits.

\section{References}

[1] C. Archetti, L. Bertazzi, and M.G. Speranza. Reoptimizing the 0-1 knapsack problem. Discrete Applied Mathematics, 158(17):1879-1887, 2010.

[2] E. Balas. Facets of the knapsack polytope. Mathematical Programming, 8:146-164, 1975.

[3] T. Belgacem and M. Hifi. Sensitivity analysis of the optimum to perturbation of the profit of a subset of items in the binary knapsack problem. Discrete Optimization, 5:755-761, 2008 .

[4] R.E. Burkard and U. Pferschy. The inverse-parametric knapsack problem. European Journal of Operational Research, 83:376-393, 1995.

[5] R.S. Dembo and P.L. Hammer. A reduction algorithm for knapsack problems. Methods of Operations Research, 36:49-60, 1980.

[6] R. Fukasawa and M. Goycoolea. On the exact separation of mixed integer knapsack cuts. Math. Program., Ser. A, 128:19-41, 2011.

[7] H.J. Greenberg. An annotated bibliography for post-solution analysis in mixed integer programming and combinatorial optimization. In D.L. Woodruff, editor, Advances in Computational and Stochastic Optimization, Logic Programming, and Heuristic Search, pages 97-148. Kluwer, 1998.

[8] P. Hansen and J. Ryan. Testing integer knapsacks for feasibility. European Journal of Operational Research, 88:578-582, 1996.

[9] M. Hifi, 2007. Personal correspondence.

[10] M. Hifi and H. Mhalla. Sensitivity analysis to perturbations of the weight of a subset of items: The single knapsack case study. Electronic Notes in Discrete Mathematics, 36:439_ 446, 2010.

[11] M. Hifi, H. Mhalla, and S. Sadfi. Sensitivity of the optimum to pertubations of the profit or weight of an item in the binary knapsack problem. Journal of Combinatorial Optimization, 10:239-260, 2005.

[12] H. Kellerer, U. Pferschy, and D. Pisinger. Knapsack Problems. Springer, 2004. 
[13] D. Klein and S. Holm. Integer programming post-optimal analysis with cutting planes. Management Science, 25:64-72, 1979.

[14] L. N. Kozeratskaya, T. T. Lebedeva, and I. V. Sergienko. Stability, parametric, and postoptimality analysis of discrete optimization problems. Cybernetics and Systems Analysis, 19:522-535, 1983.

[15] S. Martello, D. Pisinger, and P. Toth. Dynamic programming and strong bounds for the 0-1 knapsack problem. Management Science, 45:414-424, 1999.

[16] S. Martello and P. Toth. An upper bound for the zero-one knapsack problem and a branch and bound algorithm. European Journal of Operational Research, 1:169-175, 1977.

[17] M. Monaci and U. Pferschy. On the robust knapsack problem. SIAM Journal on Optimization, 23(4):1956-1982, 2013.

[18] M. Monaci, U. Pferschy, and P. Serafini. Exact solution of the robust knapsack problem. Computers \& OR, 40:2625-2631, 2013.

[19] D. Pisinger. A minimal algorithm for the 0-1 knapsack problem. Operations Research, 45:758-767, 1997.

[20] D. Pisinger. Core problems in knapsack algorithms. Operations Research, 47:570-575, 1999.

[21] A. Plateau and G. Plateau. Reoptimization for 0-1 knapsack problems. In Proc. Congreso Latino-Iberoamericano de Investigacion Operativa, Rio de Janeiro, Brazil, September 24282012.

[22] J. Seelander. Einige bemerkungen zur bestimmung von stabilitetsbereichen in der reinganzzahligen linearen optimierung. Math. Operationsforsch. Statistic, Ser. Optimization, 11:261-271, 1980.

[23] F. Vanderbeck. Computational study of a column generation algorithm for bin packing and cutting stock problems. Math. Program., Ser. A, 86:565-594, 1999. 


\section{Appendix A - Illustration of the Main Theorems}

Fig. 2 shows two knapsack instances and the corresponding dynamic programming tables $z_{j}(d)$. Both instances have $n=4$ and $c=7$. We want to find the tolerance limits for the last item $k=4$.

In the left instance we find $z=8, r=0$ hence $P=3, W=6$. Since $x_{4}^{*}=0$, Theorems 1 and 2 give us the tolerance limits $0 \leq p_{4}^{\prime} \leq 3$ and $1 \leq w_{4}^{\prime}$.

We could also reach these limits from the dynamic programming. For $p_{4}^{\prime}$ we note that $z_{4}=$ $\max \left\{z_{3}(c), z_{3}\left(c-w_{4}\right)+p_{4}^{\prime}\right\}=\max \left\{8,5+p_{4}^{\prime}\right\}$, where the first term is chosen as long as $p_{4}^{\prime} \leq 3$.

For $w_{4}^{\prime}$ we note that $z_{4}=\max \left\{z_{3}(c), z_{3}\left(c-w_{4}^{\prime}\right)+p_{4}\right\}=\max \left\{8, z_{3}\left(c-w_{4}^{\prime}\right)+1\right\}$, where the first term is chosen as long as $z_{3}\left(c-w_{4}^{\prime}\right) \leq 7$, which by inspection in $z_{3}(d)$ can be seen to hold for $c-w_{4}^{\prime} \leq 6$.

In the right instance we find $z=11, r=3$ and $P=3, W=5$. Since $x_{4}^{*}=1$, Theorems 1 and 2 give us the tolerance limits $p_{4}^{\prime} \geq 3$ and $2 \leq w_{4}^{\prime} \leq 5$.

Using dynamic programming we note for $p_{4}^{\prime}$ that $z_{4}=\max \left\{z_{3}(c), z_{3}\left(c-w_{4}\right)+p_{4}^{\prime}\right\}=\max \{8,5+$ $\left.p_{4}^{\prime}\right\}$, where the second term is chosen as long as $p_{4}^{\prime} \geq 3$.

For $w_{4}^{\prime}$ we note that $z_{4}=\max \left\{z_{3}(c), z_{3}\left(c-w_{4}^{\prime}\right)+p_{4}\right\}=\max \left\{8, z_{3}\left(c-w_{4}^{\prime}\right)+6\right\}$, where the second term is chosen as long as $z_{3}\left(c-w_{4}^{\prime}\right) \geq 2$. However, this is not sufficient to ensure that the current optimal solution $x^{*}=(1,0,0,1)$ remains optimal, since choosing e.g., $c-w_{4}^{\prime}=6$ (i.e., $\left.w_{4}^{\prime}=1\right)$ will result in an optimal solution $x^{*}=(0,1,0,1)$ with value $z=13$. As observed in the proof of Theorem 2, the current optimal solution $x^{*}=(1,0,0,1)$ remains optimal if and only if $z_{3}\left(c-w_{4}^{\prime}\right)=z_{3}\left(c-w_{4}\right)=5$, which by inspection in $z_{3}(d)$ can be seen to hold for $2 \leq c-w_{4}^{\prime} \leq 5$.

$$
\begin{array}{|r|llll|}
\hline j & 1 & 2 & 3 & 4 \\
\hline p_{j} & 5 & 7 & 3 & 1 \\
w_{j} & 2 & 6 & 5 & 2 \\
\hline
\end{array}
$$

\begin{tabular}{|r|lllll|}
\hline$d \backslash j$ & 0 & 1 & 2 & 3 & 4 \\
\hline 0 & 0 & 0 & 0 & 0 & 0 \\
1 & 0 & 0 & 0 & 0 & 0 \\
2 & 0 & 5 & 5 & 5 & 5 \\
3 & 0 & 5 & 5 & 5 & 5 \\
4 & 0 & 5 & 5 & 5 & 6 \\
5 & 0 & 5 & 5 & 5 & 6 \\
6 & 0 & 5 & 7 & 7 & 7 \\
7 & 0 & 5 & 7 & 8 & 8 \\
\hline
\end{tabular}

$$
\begin{array}{|r|llll|}
\hline j & 1 & 2 & 3 & 4 \\
\hline p_{j} & 5 & 7 & 3 & 6 \\
w_{j} & 2 & 6 & 5 & 2 \\
\hline
\end{array}
$$

\begin{tabular}{|r|rrrrr|}
\hline$d \backslash j$ & 0 & 1 & 2 & 3 & 4 \\
\hline 0 & 0 & 0 & 0 & 0 & 0 \\
1 & 0 & 0 & 0 & 0 & 0 \\
2 & 0 & 5 & 5 & 5 & 6 \\
3 & 0 & 5 & 5 & 5 & 6 \\
4 & 0 & 5 & 5 & 5 & 11 \\
5 & 0 & 5 & 5 & 5 & 11 \\
6 & 0 & 5 & 7 & 7 & 11 \\
7 & 0 & 5 & 7 & 8 & 11 \\
\hline
\end{tabular}

Figure 2: Two knapsack instances and the corresponding dynamic programming tables $z_{j}(d)$. In both instances $n=4$ and $c=7$. The two instances only differ with respect to $p_{4}$. In the left instance, $z^{*}=8$ and $x^{*}=(1,0,1,0)$. In the right instance, $z^{*}=11$ and $x^{*}=(1,0,0,1)$. 


\section{Appendix B - Special Cases}

In this section we consider some special cases in which the tolerance intervals can be identified easily as shown by Hifi et al. [11]). First, we need some definitions: We define the residual capacity $r \geq 0$ of a KP as

$$
r=c-y\left(\mathrm{WKP}\left[z^{*}\right]\right),
$$

where $z^{*}$ is the optimal solution value to KP. If several solutions to KP have the same solution value $z^{*}$ the residual capacity $r$ is the largest possible free space among all optimal solutions.

Lemma 1 (Theorem 2.1 in Hifi et al. [11]) If $x^{*}$ is an optimal solution for $K P$, and $\left(\Delta p_{k} \geq 0\right.$ and $\left.x_{k}^{*}=1\right)$ or $\left(\Delta p_{k} \leq 0\right.$ and $\left.x_{k}^{*}=0\right)$, then $x^{*}$ is an optimal solution for $K P_{\Delta p_{k}}$.

Lemma 1 states that $x^{*}$ remains optimal for $\mathrm{KP}_{\Delta p_{k}}$ if $x_{k}^{*}=1$ and $p_{k}$ increases, or if $x_{k}^{*}=0$ and $p_{k}$ decreases. The tolerance limits are hence upward (downward) unlimited in these cases as long as the profit $p_{k}+\Delta p_{k}$ remains nonnegative (since we have defined the KP as having nonnegative profits.)

Lemma 2 (Theorem 3.1 in Hifi et al. [11]) If $x^{*}$ is an optimal solution for $K P$, and $\left(0 \leq \Delta w_{k} \leq r\right.$ and $\left.x_{k}^{*}=1\right)$ or $\left(\Delta w_{k} \geq 0\right.$ and $\left.x_{k}^{*}=0\right)$, then $x^{*}$ is an optimal solution for $K P_{\Delta w_{k}}$.

Lemma 2 states that $x^{*}$ remains optimal for $\mathrm{KP}_{\Delta w_{k}}$ if $x_{k}^{*}=0$ and the weight is increased. Moreover if $x_{k}^{*}=1$ and the weight is increased up to the residual capacity, the solution $x^{*}$ remains optimal. 